\title{
Clinical Investigation of Multiple Drug Resistant Tuberculosis in the Tamale Metropolitan Area
}

\author{
Dr. Abdallah Yahaya Idrissu* \\ Senior Lecturer, University for Development Sudies, Uds, Dept. of Medicine and Allied Science, Clinician, \\ Tamale Teaching Hospital, Regional Tb Cinician, Tamale
}

\begin{abstract}
*Corresponding Author: Dr. Abdallah Yahaya Idrissu, Senior Lecturer, University for Development Sudies, Uds, Dept. of Medicine and Allied Science, Clinician, Tamale Teaching Hospital, Regional Tb Cinician, Tamale, Ghana
\end{abstract}

\begin{abstract}
After a long period of decline, tuberculosis (TB) incidence and mortality in the Tamale Metropolis rose dramatically in the 1990s and peaked in $2000^{s} .{ }^{1}$ During the same period, the proportion of notified TB patient's, cured by therapy; precipitously fell from 90\% in 1990 to an estimated $72 \%$ in 2011. Despite the introduction and gradual uptake over the past decade of the DOTS strategy, treatment success rates have remained consistently low; even though case notifications have declined. WHO attributes these high failure rates to drug resistance, high rates of default and death among patients receiving DOTS. We conducted a retrospective cohort study of newly detected smear - and/ culture-positive. TB patients aged over 17 who were notified under DOTS and began TB treatment during the period from 1 January 2009 to 31 December 2010. We excluded patients who were admitted to psychiatric hospitals, were in prison, died within one month of beginning therapy or did not live within Tamale city limits. Individuals and programmatic risk factors as well as outcomes were assessed by reviewing patients' charts and TB treatment records: through a TB database set up by the NTP (National TB Programme). We assessed risk factors for non-adherence, default and the development of MDR during therapy. In an earlier study; we reported the causes of death of patients undergoing DOTS treatment in Tamale Hospital, from January 2001 to December, 2004.
\end{abstract}

We observed a $9.6 \%$ death rate during TB treatment-due, not only to TB but also comorbidity conditions such as alcoholism and cardiovascular disease. We also found that both alcoholism and late presentation contributed substantially to mortality. Based on our findings, we proposed several specific interventions that may improve treatment outcomes and reduce the acquisition of drug resistance in patients undergoing TB therapy in this setting as follows:

1. Intensive education and training of staff is necessary to improve compliance and adherence to Direct Observatory Treatment (DOTS);

2. Mass education is obligatory to attract TB patients to come to the hospital for screening and early intervention;

3. TB management in the hospital will have to be patient centered treatment; as requested by the World Health Organization in their millennium goals, at Almata in 1978.

4. Enablers' package will have to be improved to reduce defaulting and absconding,

5. All this will lead to reduction of acquisition of multiple drug resistance, morbidity and mortality.

\section{Study Area:}

Tamale is the capital town of Northern Ghana. Its population in the year 2010 was 360579. Tamale has been described as the fastest growing city in South Saharan Africa. Poverty and low education level are major developmental barriers to this area. Tamale is a traditional community with markedly high illiteracy level. This makes the spread of Tuberculosis $(T B)$ and other communicable diseases very easy; thus making the control of diseases really difficult. The increase in population leads to increase in prevalence of Tuberculosis (TB).Tamale Teaching Hospital is a referral hospital for the three Northern Regions of Ghana (that is Upper East, Upper West and Northern Regions).

\section{INTRODUCTION / EPIDEMIOLOGY}

Tamale is the capital town of Northern Region of Ghana. Its population was 360579 according to the (2010 census). Tamale has been described as the fastest growing city in South Saharan Africa by UNICEF. Poverty and low educational levels are major developmental barriers to this area. Tamale is 
a traditional community with markedly high illiteracy level. This makes the spread of Tuberculosis (TB) and other communicable diseases very easy; thus making the control of diseases very difficult. The increase in population rather leads to increase in prevalence of Tuberculosis (TB).

Tuberculosis (TB) is an old disease but one which health workers all over the world are finding harder and harder to control. The World Health Organization (WHO) in 1993 declared TB a global emergency in recognition of this disease a public health problem. In August 2005, TB was declared an African emergency. About one-third of the world's population is infected by mycobacterium, TB.

Worldwide, in 2000 , there were about 8.7 million cases of TB diseases with 1.9 million deaths. Mycobacterium Tuberculosis kills more people than any single infectious disease agent. Death from TB accounts for twenty-five percent of all avoidable deaths, in developing countries.

Of most cases, in some developing countries, $75 \%$ are in the economic productive age (15-50) years. HIV is fueling TB epidemic; In the year $2000,1 / 3^{\text {rd }}$ of HIV infected persons worldwide (about 13 million people) were also infected with $\mathrm{M}>$ tuberculosis, $50 \%$ will become sick with TB during their life time; $10 \%$ will become sick per year. Thus, the prevalence of HIV in a community has an important effect on the incidence of TB; HIV poses as fuel to the epidemy of tuberculosis in any community.

According to Professor Hesse, without treatment, 50\% patients with pulmonary TB will die within 5 years whiles $25 \%$ remains with chronicinfectious TB; the other $25 \%$ will spontaneously recover and be healthy as a result of their strong immunity.

It is estimated that Ghana has 125 smear positive pulmonary TB cases per 100000 population and 281 of all types of TB casesper 100.000populations per year. This means that with a population of about 25 million, we should expect about 25,000 smear positive pulmonary TB cases and 56.000 new TB cases of all types. Every year, with about 12,000 deaths.

However, only about a fifth of these cases $(12,000)$ are reported in this country every year; according to (2007-2010) TB - CAP reports, by Rehab Chimzizi. This is because of the following reasons:

- Many people with TB do not report to health facilities;

- Those who report to our health facilities are not diagnosed as TB (miss diagnosed);

- Not all diagnosed cases at the health facilities are captured by the disease surveillance system; and

- Most TB patients are adults in the most productive age group and this adversely affects the national and family economy.(Reports from TB Control Assistance Program (TB CAP) Ghana (2007-2010)

Although Ghana is not one of the 22 TB high burden countries, the disease remains a major public health problem in the country. The WHO estimates that Ghana should be reporting at least 45,000 TB cases on an annual basis but only one third of the cases are detected. This means that many more undiagnosed TB in the communities are continuously transmitting the disease to susceptible people.

\subsection{Rationale}

- Though a major Public health burden, no surveillance has been done in the Tamale Metropolis as yet.

- TB needs to be controlled because it has a major public health importance to the Tamale Metropolitan area. Success in treating resistant TB will increase the treatment success rate in the Tamale metropolis.

- Multiple drug resistance tuberculosis (MDR)-TB is a treat to TB control in Ghana.

\subsection{Objectives}

The objectives of this study are as follows:

- To verify the presence of multiple drug resistance in the Tamale Metropolitan area;

- To identify barriers to treatment success in Tamale Metropolitan area; 
- To outline the causes of treatment failure of tuberculosis in the Tamale metropolitan area; and

- To recommend more appropriate methods for the control of TB at the Tamale Metropolis

\section{LITERATURE REVIEW}

After studying TB Treatment in a High HIV-Prevalence Setting in Southern Africa, PLoS One. 2009; 4(9): e7186.r recorded the stated information in the 2009 WHO bulletin as written below; multiple drug resistant tuberculosis (MDR TB) in Africa may be more prevalent than previously appreciated. Factors leading to development of drug resistance need to be understood to develop appropriate control strategies for national programs. Estimates of MDR TB rates for 39 out of 46 countries were gathered, in Africa. The relationship between MDR TB rates and independent factors was analyzed by using correlation and linear regression models. Their findings indicated that drug resistance surveys in Africa are critically needed. They suggested that MDR TB rates must be assessed in countries without these surveys. They wrote that even in countries that have conducted a drug resistance survey; a new survey will determine evolution of drug resistance rates. They also found no correlation between high MDR rates and TB incidence, HIV/TB co-infection rates, or year of introduction of rifampin. Results show that the retreatment failure rate was the most predictive indicator for MDR TB. Current category II drug regimens may increase MDR TB. According to Prof. Gerald Friedland South Africa has the highest rates of XDR in Africa. He mentioned this in his lecture in Ghana On $22^{\text {nd }}$ January 2010.

In the year 2004 and 2009, M Kawakami, S Takikawa, and K Kawakami did a clinical investigation on multiple drug resistant tuberculosis in Japan, and found that in drug therapy group there were more cases with resistance to 5 drugs, resistance to levofloxaciline (LVFX) and Kanamicine (KM) in those who died of tuberculosis than in non-tuberculosis death cases.

They concluded that resistance to fight drugs; resistance to LVFX, and resistance to KM were contributory factors of tuberculosis death. This is typical example of Extensive Drug-Resistant Tuberculosis (XDR-TB) as defined in Oct 2006. From this same clinical investigation of MDR, they observe that patient's operability was one of the factors associated with a good prognosis.

In the year 2005 IF Hesse, FA Bonsu, KK Addo, and MJ Newman; undertook a survey at the chest clinic of the Korle-Bu Teaching Hospital in Accra. They found that the level of drug resistance in mycobacterium isolate from previously treated cases in Ghana was not known; all though the drug resistant tuberculosis threatened efforts to control the disease. Eighteen isolates of mycobacterium were identified from 28 failed treatment cases. After culture and sensitivity of mycobacterium isolates from cases of pulmonary tuberculosis, classified as treatment failures, in a Teaching Hospital were analyzed.

This group of doctors from the Korle Bu Teaching Hospital therefore concluded that "contentious drug resistance monitoring must be instituted as part of the tuberculosis control program".

On January 222010 Professor Gerald Friedland, a TB expert from the United State, cautioned Ghana of MDR-TB. In his lecture under the theme; "confronting multiple drug resistant tuberculosis (MDR)-TB and extensive drug resistant tuberculosis (XDR)-TB in sub-Sahara Africa". He said there was still much more to be done in ensuring more case detection and treatment of the disease. $\mathrm{He}$ further explained that, though Ghana had not yet recorded any extensively drug resistant tuberculosis (XDR)-TB, as compared to South Africa; that had the highest rate of XDR, "You need not wait until you get to that stage".

The national tuberculosis control program's annual reports, in 2003 recorded a primary drug resistance rate of $23.5 \%$ in Ghanaian TB; this ranks Ghana among those African countries with high prevalence of drug-resistant TB. Prof Friedland noted that the widespread of prevalence rate in South Africa had been so serious that neighbouring countries like Bostwana, Mozambique, Lesotho, Swaziland, Namibia, and Zimbabwe had been affected, with no denominator. And that access to care was also limited. He said what could be done in the short term; to prevent or reduce the impact of MDR and XDR-TB in Ghana was; "rapid and massive infusion of resources for TB to decrease the generation of acquired cases".

As of Oct 2006, "extensively drug resistant tuberculosis (XDR-TB) was defined as MDR-TB that is resistant to quinolones and other second inline drugs such as kanamycin, capremcin, or amikacin. The old case definition of XDR-TB is MDR-TB that is also resistant to three or more of the six classes of 
second-line drugs. This definition should no longer be used but included here because many older publications refer to it. The epidemiology of XDR-TB is currently not well studied; it is believed that $\mathrm{XDR}-\mathrm{TB}$ does not transmit easily in healthy populations, but is capable of coursing epidemics in populations which are already stricken by HIV and therefore more susceptible to TB infection.

Review of the worldwide situation and the WHO/IUATLD Global Surveillance Project, by David L. Cohn, Flavia Bustreo and Mario C.R in 1985 and 1994, revealed that: the emergence of strains of mycobacterium tuberculosis that resistant to antimycobacterial agents is a worldwide problem whose global magnitude is not well described. They reviewed and tabulated 63 surveys of resistance to antituberculous drugs. Rates of primary resistance to isoniazid, administered as a single agent, ranged from 0 to $16.9 \%$ ( median rate $4.1 \%$ ); to streptomycin, $0.1 \%-23.5 \%$ (median, $3.5 \%$ ); to rifampicin, 0 $3.0 \%$ (median, $0.2 \%$ ); and to ethambutol, $0-4.2 \%$ (median, $0.1 \%$ ).

They concluded that; having developed basic recommendations for antituberculous drug resistance surveillance, created an SRL Network, and provided software for data analysis, WHO and IUATLD will continue to support drug resistance surveillance projects in various countries. These will result in a comprehensive overview of the drug resistance situation in many countries; more accurate information and better trend data than are currently available. Policy recommendations to decrease or stabilize emerging antituberculous drug resistance are ultimately expected to result from this global effort.

In October 2007 Matteelli A, Migliori GB, Cirillo D, Centris R, Girard Eand Raviglion M; wrote in a journal "Expert rev anti infect ther". That the emergence of multidrug-resistant (MDR)- TB and, more recently, of extensively drug-resistant (XDR)- TB is a real threat to achieve TB control and elimination. Over 400,000 new cases of MDR-TB occur each year and, although their number is currently unknown, XDR cases are recognized in every setting where there has been the capacity to detect them. The long-term vision for the full control of MDR-TB requires the scalling-up of culture and drugs-susceptibility testing capacity, which is very limited in disease-endemic countries, and the expanded use of high-technology assays for rapid determination of resistance. MDR cases are treatable and well-designed regimens, largely based on second-line anti-TB drugs, can considerably improve through the introduction of less toxic and more powerful drugs, thus reducing duration of treatment and tolerability. This is of unmost importance for XDR-TB cases. The prevalence of MDRTB are inversely correlated with the quality TB control and the proper use of the second-lone anti- TB drugs. Adherence to proper standards of care and control is imperative and a top priority of all TB control efforts. However, the risk of an uncontrollable epidemic of MDR- and XDR- TB is real considering current levels of financing and commitment to care.

\section{METHODS}

\subsection{Setting and Programme Description}

We conducted this study in the Tamale Metropolis, where the incidence and mortality rates of TB in 2009 were 109.3 and 8.3 per 100.000, respectively. Rates of MDR in Tamale Teaching Hospital were among the highest reported worldwide; MDR among newly diagnosed patients rose from $6.5 \%$ in 2009 to $12.1 \%$ by 2011 . Tamale Teaching Hospital was one of the first in the Northern Region of Ghana to implement the DOTS strategy.

Tamale Teaching Hospital (TTH) oversees diagnosis, treatment and reporting of adult patients with TB. Suspects undergo sputum smear microscopy and culture at the time of diagnosis. Those who are culture positive also undergo drug sensitivity testing to isoniazid, rifampicin, ethambutol, streptomycin and kamamycin. Susceptibility is determined using the absolute concentration method on Lowenstein-Jensen medium, based on the following drug concentrations: isoniazid $1 \mu \mathrm{g} / \mathrm{ml}$, ethambutol $5 \mu \mathrm{g} / \mathrm{ml}$ and streptomycin.

Patients diagnosed with active TB are treated according to WHO recommendations. ${ }^{5}$ Those with multidrug-resistant TB (MDR- TB) are switched to an individualized regimen based on the drug resistance profile. Treatment is offered three ways: under direct supervision in an inpatient setting, at one of three outpatient clinics or through home-based care. Patients receive drugs daily in each of the outpatient settings. Home-based care is provided for those who are unable to attend outpatient clinics, with nurses delivering drug directly to the patients. Some patient self-administered drugs during weekends and holidays, and a small proportion self-administered over half of their medications. All 
patients treated in ambulatory settings. Travel expenses are not provided for patients who have no public transport services. Patient undergoing TB treatments are assessed with repeat sputum smear, culture, and drug-sensitivity testing (DST) in months 2,3 and 5 as well as at the end of treatment and at six-month intervals thereafter.

\subsection{Exposure Assessment}

The following information collected routinely for all patients undergoing TB therapy were recorded under the DOTS: age, gender, address, history of previous TB treatment, clinical signs at presentation, date of diagnosis, all sputum-smear results, all culture results, and all drug-sensitivity profiles, number of missed doses, dates of missed doses, date of end of treatment, date of default, date of death, comorbidities including HIV, employment status at beginning of treatment, history of previous incarceration and diagnosis of chronic alcoholism/smoking. We classified patient' proximity to their assigned clinic on the basis of their home address and the accessibility of public transport. Patients were classified as having co-morbidities potentially associated with side effects if they reported renal insufficiency, liver disease, diabetes mellitus, gastric ulcers and management cholecystitis.

\subsection{Outcome Assessment}

We classified patients as non-adherent if they missed more than $20 \%$ of the prescribed doses during the treatment period recommended by WHO. In a sensitivity analysis, we identified patients who missed more than $50 \%$ of their prescribed doses. Treatment outcomes, including default, were classified according to WHO guidelines. ${ }^{6}$ Patients were classified as having acquired MDR during or subsequent to therapy if they were sensitive to either isoniazid or rifampicin on their first DST but were noted to be resistant to both agents on any later DST.

\subsection{Data Analysis}

For univariate analyses of non-adherence and default, we used logistic regression to estimate odds ratios (ORs) and 95\% confidence intervals (CIs). The Mantel-Haenszel $x^{2}$ method or Fisher's exact test was used to calculate p-values. Statistical tests were two-sided. We used separate logistic regression models to perform multivariate analyses of the outcomes, adherence and default. The multivariate model included relevant variables with p-values less than 0.2

Kaplan-Meier survival analysis was used to estimate the time from initiation of therapy to acquisition of MDR -TB. For patients who did not reach the end of study period, the data were censored at the time of their last DST. The MDR acquisition time was taken as the mid-point between the last DST without MDR and the first DST with MDR. The log rank test was used to compare time to MDR between strata. The Cox proportional hazards model was used for multivariate analysis. In a subanalysis, we also assessed risk factors for early (within 4 months of treatment initiation) and late (6 months after treatment initiation) acquisition of MDR. Patients who acquired early MDR were excluded from the analysis of risk factors for late MDR. Analyses were performed using Strata (version 9.0) and SAS (version 9.1) software.

\section{RESUlts}

Of the 260 civilian adult patients enrolled in the DOTS treatment programme during the study period, 8 died during the first month of therapy and 12 had missing treatment records (Fig. 1). The remaining 234 patient were included in the analysis of non-adherence and default; there were 148 men and 89 women and the mean age was 40. Primary MDR was found in $20(8.4 \%)$ of the patients, and 82 $(34.5 \%)$ were found to be resistant to at least one drug at the time of diagnosis. Excluded patients were more likely to be male, unemployed, homeless and substance abusers. Among the 137 patients included in the study, 20 had MDR on initiation of therapy and 10 had missing initial DSTs; the remaining 107 participants were included in the analysis of MDR acquisition. All patients were HIV tested, but since only two were found to be HIV-positive we did not include HIV in our subsequent analyses.

Treatment outcomes were presented in Table 1. More than half of those who died (8/14) did so within the first 4 months of treatment. The overall mortality of patients undergoing DOTs therapy for TB is underestimated, as the data presented in Table 1 exclude results on these patients. Twenty-one (8.8\%) of the patients in our cohort defaulted on therapy and 37 (15.6\%) took fewer than $80 \%$ of their 
observed prescribed doses. Fifteen patients (6.3\%) acquired MDR during the study period, seven during the course of treatment and eight during post-treatment follow-up.

Baseline characteristics of those who defaulted or were non-adherent are given in Table 2. In a multivariate model, herbal use was identified as the only factor that was strongly associated with nonadherence with odds ratios for baseline alcohol dependence-4.38 (95\% CI: 1.58-12.60); reported smoking use in a patient during therapy - 6.35 (95\% CI: 2.27-17.750; the adjusted odds ratio of nonadherence for those with any kind of substance abuse was 7.30 (95\% CI: 2.89-18.46).

Substance abuse (herbal abuse) was also strongly associated with default, with an odds ratio of 15.57 (95\% CI: 3.46-70.07) among those with baseline alcoholism and 5.14 (95\% CI: 0.87-30.25) for those with reported alcohol use. Patients with any form of substance abuse had an adjusted odds ratio for default of 11.20 (95\% CI: 2.55-49.17). When this analysis was repeated, excluding patients for whom more than $40 \%$ of dose were self-administered, the odds ratios changed by less than $20 \%$. This shows that non-adherence was associated with poor treatment outcomes (OR: 2.43, 95\% CI: 1.05-5.53).

Sputum-smear positivity was the only factor associated significantly with baseline MDR in both univariate analysis $(\mathrm{OR}=2.4,95 \% \mathrm{CI}$ : $1.04-5.57)$ and multivariable logistic regression model that included age and substance abuse ( $\mathrm{OR}=3.28,95 \% \mathrm{CI}$ : 1.24-8.68). Factors associated significantly with MDR acquisition in a univariate analysis included substance abuse, hospitalization (both at initiation of treatment and later in the course of therapy) and failure to self-administer therapy (Figs. 2, 3, 4; and Table 4. Patients who received treatment in the hospital setting had a substantially higher risk of developing MDR-TB than those whose treatment was confined to the outpatient sector. This was true for those who began DOTs treatment in the hospital setting (adjusted hazard ratio, HR: 6.34; $\mathrm{p}=0.02$ ) and those who were hospitalized only later in their treatment course (adjusted HR: 6.26; $\mathrm{p}=0.04)$.

Notably, non-adherence was not a risk factor for either early or late acquisition of MDR. This finding remained true when we conducted a sensitivity analysis in which patients were classified nonadherent if they missed $40 \%$ or more of their prescribed doses.

Patients' records were reviewed and follow up was done through telephone communication with patients and their relatives or treatment supporters. Also with the help of addresses and descriptions. Patients were visited in their homes for reassessment, interview and physical examination.

Sputum samples were taken from patients where hospitals or their homes for microscopic analysis. Patients with sputum positive results were identified and labeled as treatment failure.

Death rate and treatment success continues to improve as time went on.DOTS were adhered to and our clinical methods focused on patients centered techniques as demanded by the WHO in their millennium goals in Almata in 1978.

\section{DISCUSSION}

In this study of non-adherence, default and acquisition of MDR among TB patients in Tamale; herbal use and in-hospital care were identified as two potential obstacles to effective treatment. These results suggest that DOTS program might be more likely to achieve TB control targets if they include interventions aimed at improving adherence by diagnosing and treating substance abuse concurrently with standard TB therapy. They also raised the possibility that some patients with apparent drugsensitive diseases may also be infected with drug-resistant strains that are unmasked upon initiation of therapy. Some patients might also have been reinfected with drug-resistant strains in the hospital setting, a possibility which emphasizes the need for effective infection-control measures within facilities that care for patients with active diseases.

Despite the implementation of DOTS programs and the provision of extensive social services to patient undergoing TB therapy, non-adherence and default continued in a substantial proportion. These patients were burdened with a wide array of social and medical problems: many were unemployed, while some had been in serious poverty and other social burdens; depression and significant co-morbid conditions. Despite the use of herbs, alcoholism and smoking were the only independent risk factors for non-adherence and default that were identified. These findings echo those of numerous previous studies that found substance abuse to be the single major factor associated most 
strongly with non-compliance with TB regimens .Our results also agrees with those previous studies from the World Health Organization (WHO) bulleting in 2000, 2005,2009; findings that nonadherence has important adverse effects on the outcomes of TB treatment. All poor outcomes experienced in our cohort occurred among the $16 \%$ of patients who did not adhere to therapy.

Despite the clear need for new approaches to this problem, up to date, there has been relatively little research on treatment options for patients with chronic infectious diseases and concomitant herbal misuse. Program that have explicitly offered patient treatment for abuse, generally have demonstrated better outcomes than "unexpanded" DOTS program. some even achieve very high cure rates among patient populations in which alcoholism or herbal use was predominant.

This failure has at least three possible explanations. The first is the reluctance to tinker with the specialized "vertical" DOTS approach; given its success in improving case completion and cure rates in developing and less-developed countries over the past two decades. Secondly, closely related to this are the numerous obstacles faced by multidisciplinary approaches to research and patient care, including the lack of a shared language and space among care providers from different specialties and mutual lack of knowledge of other treatment approaches.

Thirdly, often the care of TB patients and those with substance-use disorders is relegated to highly specialized practitioners; this offers little opportunity for meaningful interaction or exchange between disciplines. Finally, until recently many physicians without specific expertise in managing alcohol disorders and smoking misuse have assumed that these conditions' treatment are too complex and intensive to be carried out simultaneously with the treatment of another complex disease. However, recent evidence suggests that brief interventions, social skills training, behavior contracting and pharmacotherapy are among the most effective approaches for treatment of substance-use disorders. These data raise the possibility that integrated management of these most vulnerable TB patients may be within the reach of a unified TB care facility.

Our study also suggests that non-adherence did not contribute to either the early or late occurrence of MDR among patients receiving DOTS in this setting. We considered several other possible explanations for the observation that a group of adherent patients developed several others within 24 months of initiating therapy. First, we speculated the MDR acquisition might be associated with disease severity, which might in turn be linked to hospitalization. Since the number of new mutations that code for drug resistance will be a function of the bacterial load, it follows that those with a greater disease burden would be at higher risk of developing these mutations. Secondly having adjusted for disease severity, by controlling the presence or absence of cavitation and sputum-smear status, we found that these markers of disease severity were strongly correlated with early acquisition of MDR but not associated with late acquisition. These data suggest that these patients may harbor different strains of Mycobacterium TB, some of which may be drug-resistant.in these mixed infections, standard short-course therapy may have unmasked the drug-resistant strain population by suppressing the previously dominant drug-sensitive strain. Indeed, van Rie et al;2004,have described this mechanism in a high-burden population in South Africa. Thirdly in that study, adherence to a first-line drug therapy was shown to select for a resistant population, while non-adherence led to re-emergence of the drug-susceptible strain. We also assessed the possibility that patients who developed MDR did so through "amplification" of exiting drug resistance. While this mechanism may have accounted for MDR acquisition in some cases, eight of the thirteen hospitalized patients with this outcome had fully susceptible disease on initiation of therapy.

Finally, we considered the possibility that some of these patients developed MDR-TB as a result of reinfection with a drug-resistant strain of TB. Reinfection of patients on therapy for drug-sensitive diseases had been described in several different high-incidence settings and has been associated with nosocomial transmission(A. Kruuner) 2001. Usually, MDR -TB patients in the Tamale Metropolitan Area are not placed on respiratory precautions in the hospitals or clinics where they receive care. This is because our doctors and nurses do not understand the importance of respiratory precautions so there is opportunity for further spread of drug-resistant strains among patients receiving therapy for drugsensitive disease. The finding that substance abuse was a risk factor for late occurrence of MDR also raises the possibility that these patients are at higher risk of exposure to drug-resistant disease or are more susceptible to Reinfection than other patients. Future studies on the association between 
adherence and development of MDR would benefit from molecular typing of sequential isolates in patient undergoing therapy.

As shown in the graph, detection rate increased to the peak in 2008, plateauedin 2009 and then increase to the maximum at the end of 2011.This was because approach to management of TB at the Tamale Teaching Hospital complied with the WHO DOTS method whiles education to the public intensified. With the follow up to patients houses, the relatives who were sensitized came forward for screening thus, therefore leading to improvement of detection rate.

\section{CONCLuSion}

There are more unconfirmed cases of MDR at the TTH. More than 80 percent of our DOTS cases complete the treatment religiously but still come back with positive clinical signs and symptoms. Gene expect results shows smear positive results among some of our well-reviewed cases.

This shows that there are more presumed MDR cases than documented at TTH.

Barriers to treatment success rate in the Tamale Metropolitan area are caused by defaulting of patients, lack of compliance and lack of adherence to DOTS by treatment supporters and health care providers. These include:

- Lack of compliance by patients;

- Lack of adherence to DOTS by the health workers;

- Lack of training of clinical supporters; and

- Default by TB patients.

\section{RECOMMENDATION}

More presumed cases must $\mathrm{Be}$ sent out to Korle- $\mathrm{Bu}$ or nugushie for confirmation before recording. The midget machine must be well fixed for culture and sensitivity. The gene expect must be repaired for early detection and conformation of MDR.

More precise counselling must be done to prevent default of patients and lack of adherence to DOTS.

Training of clinical/treatment supporters must be well done for effective management and result

\section{ACKNOWLEDGMENT}

My sincere acknowledgement go's to the following lectures of the University for Development Studies for helping me make this work a success;

Mr. Doklah Kwame Anthony of Catholic University, ICST Department

Dr. Sam Bugri: Head of Department Community Health and Development School of Medicine and Health Sciences

Dr. D Konla: Snr lecture in Epidemiology School of Medicine and Health Sciences

Dr. Mahama Saaka: (CCEIR)

Dr. Ken Sagoe: Chief Executive Officer of Tamale Teaching Hospital

Dr. Sir, Gyadery: Dean, School of Medicine and Health Sciences University for Development Studies.

\section{REFERENCES}

[1] Kok-jensen. The prognosis of pulmonary tuberculosis in patients with abuse of alcohol. Scand J Repair Dis 1979; 51:42-8.

[2] Kruuner, SE Hoffner, H Sillastu, M Danilovits, K Levina, SB Svenson, et al., et al. spread of drugresistant pulmonary tuberculosis in Estonia. J Clin Microbiol 2001; 39: 3339-45.

[3] Pablos-Mendez, CA Knirdch, RG Barr, BH Lerner, TR Frieden. Nonadherence in tuberculosis treatment: predictors and consequences in New York City. Am J Med 1997; 102:164-70.

[4] Riviro, M Marquez, J Santos, A Pinedo, MA Sanchez, A Esteve, et al. High rate of tuberculosis Reinfection during a nosocomial outbreak of multidrug-resistant tuberculosis caused by Mycobacterium bovis strain B. Clin Infect Dis 2001; 32: 159-61. 
[5] van Rie, R Warren, M Richardson TC Victor, RP Gie, DA Enarson, et al., et al. exogenous Reinfection as a cause of recurrent tuberculosis after curative treatment. N Engl J Med 1999.

[6] A.van Rie, TC Victor, M Richardson, R Johnson, GD van der Spuy, EJ Murray, et al., Reinfection and mixed infection cause changing Mycobacterium tuberculosis drug-resistance patterns, Am J Respir Crit Care Med 2005; 172:636-42.

[7] AKSalami, PO Oluboyo. Management outcome of pulmonary tuberculosis: anine-year review in llprin. West Afr J Med 2003; 22: 114-9.

[8] C.P. Chaulk, VA Kazandjian. Directly observed therapy for treatment completion of pulmonary tuberculosis: consensus statement of the public health tuberculosis guidelines panel. JAMA 1998; 279: 943-8.

[9] E.M. Krupitsky, EE Zvartau, DA Lioznov, MV Tsoy, VY Belyaeva, et al., et al. barriers morbidity of infectious and addictive disease in St. Peterburg and the Leningrad region, Russia. Eur Addict Res 2006; 12:12-9.

[10] Global tuberculosis control-surveillance, planning and financing: Russian Federation Geneva: WHO; 2005. Available at: http://www.who.int/tb/publications/global_report/ 2005/annex1/en/index15.html

[11] J. Veen, MC, Raviglione, HL Rieder,GB Migliori, P Graf, M Graf, M Grzemska, et al., et al. standardized outcome monitoring in Europe. Recommendations of a working group of WHO AND IUATLD (Europe Region). Eur Repair J 1998: 12:505-10.

[12] J.A Cayla, JA Caminero, R Rey, N Lara, X Valles, H Galdis-Tanguis. Working group on completion of tuberculosis treatment in Spain. Int J Tuberc Lung Dis 2004; 8:458-64.

[13] K. J. Seung, IE Gelmanova, GG Peremitin,VT G olubchkova, VE Pavlova,OB Sirotkina, et al., et al., the effect of initial drug resistance on treatment response and acquired drug resistance during standardized short-course shemotherapy for tuberculosis. Clin Infect Dis 2004; 39:1321-8.

[14] M.C. Raviglione, A Pio. Evolution of WHO policies for tuberculosis control, 1948-2001. Lancet 2002; 359: 775-80.

[15] M.G Goldstein,EP Whitlock, J DePue. Planning committee of the addressing multiple behavioral risk factors in primary care project. Multiple behavioral risk factor interventions in primary care. Am J Prev Med 2004; 27:61-79.

[16] M.L. Willenbring. Integrating cares for patients with infectious, psychiatric, and substance use disorders: concepts and approaches. AIDs 2005; 19: S227-37.

[17] M.V. Shilova, C Dye. The resurgence of tuberculosis in Russia. Philos Trans R Soc Lond B Biol Sci 2001; 356:1069-75.

[18] N. Bertholet, JB Daeppen, V Wietlisbach, M Fleming, B Burnand. Reduction of alcohol consumption by brief alcohol intervention in primary care: systematic review and meta-analysis. Arch intern Med 2005; 165: 986-95.

[19] N. Schluger, C Ciotoli, D Cohn, H Johnson, wn Rom. Comprehensive tuberculosis control for patients at high risk for noncompliance. Am J Respir Crit Care Med 1995; 151: 1486-90.

[20] O. Narvskaya, T Otten, E Limeschenkova, O Graschekova, L Steklova, et al., et al. Nosocomial outbreak of multidrug-resistant tuberculosis caused by a strain of Mycobaterium tuberculosis W-Beijing family in St. Petersburg, Russia. Eur J Clin MIcrobiol infects Dis 2002; 21: 596-602.

[21] O.S. Toungoussova, G Bjune, DA Caugant, Epidemic of tuberculosis in the former Soviet Union: social and biological reasons. Tuberculosis (Edind) 2006; 86:1-10.

[22] R. Corcoran. Compliance with chemotherapy for tuberculosis. Ir Med J 1986; 79: 87-90.

[23] R. Diel, S Niemann. Outcome of tuberculosis treatment in Hamburg: a survey, 1997-2001. Int J Tuberc Lung Dis 2003; 7:124-31.

[24] R.S. Fry, Khoshnood, E Vdovicheko, J Granskaya, V Sazhin, L Shpakovskays, et. Al., Barriers to completion of tuberculosis treatment among prisoner and former prisoner in St. Peterburg, Russia. Int. J Tubec Lung Dis 2005; 9:1027-33.

[25] S. Verver, RM Warren, N Beyers, M Richardson, GD van der Spuy, MW Borgdorff, et al., et al. rate of Reinfection tuberculosis after successful treatment is higher than rate of new tuberculosis. Am J Respir Crit Care Med 2005; 171: 1430-5.

[26] T Santha, R Garg, TR Frieden, V Chandrasekaran, R Subramani, PG Gopi, et al., Risk factors associated with default, failure and death among tuberculosis patients treated in a DOTS programme in Tiruvallur District, South India, 2000. Int J Tuberc Lung Dis 2002; 6: 780-8.

[27] T.A. Mathew, TN Ovsyanikova,SS, Shin, I Gelmanova, DA Balbuena, S Atwood, et al., et al. causes of death during tuberculosis treatment in Tomsk Oblast, Russia. Int J Tuberc Lung Dis 2006; 10: 857-63.

[28] Treatment of tuberculosis: guidelines for national programmes. $3^{\text {rd }}$ ed.Geneva: WHO; 2003. 
[29] W.J. Burman, DL Cohn, CA Rietmeijer, FN Judson, JA Sbarbaro, RR Reves. Noncompliance with directly observed therapy for tuberculosis. Epidemiology and effect on the outcome of treatment. Chest 1997; 111: 1168-73.

[30] Whitlock E.P, Green CA, Polen MR. Behavioral counseling interventions in primary care to reduce risky/harmful alcohol use. Systematic evidence review. Rockville: Agency for Healthcare Research and Quality; 2004. Available at www.ahrq.gov/clinc/serfiles.htm.

[31] X. Ferrer, A Kirschbaum, J Toro, J Jadue, M Munoz, A Espinoza. Compliance with tuberculosis treatment in adults in Santiago, Chile. Bol Oficina Sanit Panam 1991; 111:423-31.

\section{AUTHORS BIOGRAPHY}

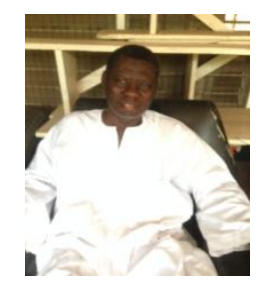

Dr. Abdallah Iddrisu Yahaya is a Ghanaian by Nationality. He is a senior lecturer at University for development studies (UDS) at the department of internal medicine. he is a consultant Physcian at the Tamale Teaching Hospital, TTH. he is also the Regional TB clinician for Northern Ghana. Medical Doctor, MSC community Health and BSc. Ziology and PHD candidate, Millar Open University.

Citation: Dr. Abdallah Yahaya Idrissu. "Clinical Investigation of Multiple Drug Resistant Tuberculosis in the Tamale Metropolitan Area ". International Journal of Humanities Social Sciences and Education (IJHSSE), vol 5, no. 6, 2018, pp. 81-90 doi: http://dx.doi.org/10.20431/2349-0381.0506011.

Copyright: (C) 2018 Authors. This is an open-access article distributed under the terms of the Creative Commons Attribution License, which permits unrestricted use, distribution, and reproduction in any medium, provided the original author and source are credited. 